\title{
Structural aspects of the liver in patients with biliary disease and portal hypertension
}

\author{
K WEINBREN, ${ }^{*}$ NS HADJIS, $†$ LH BLUMGART $\dagger$
}

From the Departments of Experimental *Pathology and †Surgery, Royal Postgraduate Medical School, London

SUMMARY Structural changes were examined in liver tissue from 28 patients with chronic bile duct obstruction in whom portal hypertension was diagnosed. Extrahepatic portal occlusion was found in three patients and cirrhosis of the liver in two. In the remaining 23 patients diffuse hepatocyte hyperplasia and portal fibrosis were observed, but a normal spatial relation between portal tracts and hepatic venous radicles was, for the most part, retained. Liver tissue was also examined from a group of 76 patients with chronic bile duct obstruction in whom there was no indication of portal hypertension but some evidence of hepatocyte hyperplasia and fibrosis. Both these features were much less extensive than the changes seen in the group of patients ostensibly suffering from portal hypertension.

The findings suggest that the combination of portal hypertension and chronic bile duct obstruction may not imply the unremitting, progressive, and irreversible changes that accompany cirrhosis because the normal vascular relations are retained. In the light of increasing experimental and clinical evidence of the resorption of collagen and the remodelling of hepatic plates it seems that the structural abnormalities in duct obstruction may substantially regress.

The development of splenomegaly and gastrooesophageal varices is regarded as a serious complication in patients with chronic bile duct obstruction, and the chances of full recovery are consequently considered to be much reduced. ${ }^{12}$ This view seems to be based on the evidence that similar clinical features are also associated with the development of cirrhosis in other forms of liver disease, in which the structural changes are considered to be irreversible. $^{34}$

Although it is not known exactly what mechanisms operate in the development of portal hypertension in chronic bile duct obstruction as the pressure measurements are not always easily interpreted in this condition, ${ }^{5}$ the generally accepted link between portal hypertension and cirrhosis in other forms of chronic liver disease is the distortion of nodular parenchyma, with its compressive effect on hepatic vessels. ${ }^{\circ}$ At the same time, the structural changes interpreted as evidence of cirrhosis in chronic bile duct obstruction are regarded as very unusual, ${ }^{78}$ but the clinical condition attributed to "secondary biliary cirrhosis" is regarded as a moderately common

Accepted for publication 13 May 1985 late change in chronic bile duct obstruction. ${ }^{12910}$

Recent observations on the lability of collagen types I and III suggest that there may be some clinical importance in defining the structural changes in chronic bile duct obstruction. ${ }^{1}$ If the profound distortion common to cirrhosis resulting from other forms of liver disease ${ }^{12}$ is also the basis of portal hypertension in chronic bile duct obstruction then resolution of the bile duct disease is unlikely to lead to an improvement in changes consequent on the portal hypertension. If, on the other hand, the hepatic changes do not include severe nodular distortion, restoration of normal haemodynamics may be possible, perhaps, with some resorption of collagen on adequate treatment of the biliary disease..$^{13}$

There are implications for both prognosis and management in the accurate structural assessment of the livers of patients in this category, and we attempt in this paper to define the hepatic histological characteristics in a group of patients with chronic bile duct obstruction and the clinical changes that represent portal hypertension. We also noted the histological changes in a second group of patients with chronic bile duct obstruction, in whom evidence of portal hypertension had not been detected by the usual clinical and imaging procedures. 


\section{Material and methods}

Sections of liver, obtained at biopsy or necropsy, were examined from 28 patients known to be suffering from chronic bile duct obstruction who showed clinical changes considered to have resulted from portal hypertension. Features interpreted as evidence of raised portal venous pressure were noted with varying frequency in the patients. The following signs formed the basis of the diagnosis of portal hypertension:

1 Raised pressure as well as clinical, imaging, and angiographic changes in three patients (two with oesophageal varices).

2 Oesophageal varices (haematemesis in five) and clinical and imaging evidence of splenomegaly in nine patients.

3 Splenomegaly, without adequate evidence of varices in sixteen patients. In these patients splenomegaly was detected in two by computed tomography, in eight by angiography, and in six by both procedures. In six patients with splenomegaly splenic tissue became available at laparotomy or necropsy, and microscopical changes associated with portal hypertension (white pulp atrophy, increased fibrosis, and iron deposition) were observed in all.

4 In 16 patients (three from group 1, eight from group 2, and five from group 3) distended veins and enlarged spleens were noted at laparotomy.

The usual clinical and imaging evidence therefore formed the basis for the diagnosis of portal hypertension in these patients, and this was strengthened by direct measurements of the portal venous pressure in three patients and clearly visible oesophageal varices in 11 patients, five of whom had also suffered episodes of haematemesis.

The lesions obstructing the bile duct consisted of fibrous strictures or tumours. Fibrous stricture caused obstruction in 19 patients, in two of whom there were associated stones. Cholangiocarcinoma was present in nine. The duration of the disease was considered to be the time since the first relevant symptoms had been noted or an operative ductal injury had been recorded (the mean duration being 4.5 years, range 6 months to 20 years). The structural features, which were recorded, include the presence of gross extrahepatic vascular obstruction, disparity in size of liver lobes, and changes on microscopy.

We examined tissues from a further 76 patients with chronic bile duct obstruction who were not regarded, either on clinical grounds or on the basis of other investigations, as having portal hypertension. We reviewed the structural changes in this group in an attempt to check whether there were major differences between these patients without portal hypertension and our main group of patients, in whom portal hypertension had been diagnosed. The disease was generally of shorter duration in this group, the mean period after onset of symptoms being 18 months (range 1 month to 4 years). Fibrous stricture was present in 33 patients, cholangiocarcinoma in 34 , and pancreatic carcinoma in nine.

\section{Results}

Extrahepatic portal venous obstruction from tumour invasion or compression was present in three of the 28 patients with the clinical features of portal hypertension. The microscopical changes in these three patients, though assessed, were not included in the microscopical analysis. A conspicuous reduction in size affected one lobe of the liver in seven of the remaining 25 patients in this group, with an apparently compensatory enlargement of the other lobe. The small lobe appeared to be fibrosed with thickened ductal structures and generally firm tissue showing grey streaks between lobules and with crowding of ductal and vascular structures in the gross specimen. The reduction of size was detected on examination by computed tomography and the crowding of vessels and ducts was also noted at angiography and cholangiography. An appreciable disparity in size between the two liver lobes was also found in eight patients without evidence of portal? hypertension; the most striking example of this was in a patient suffering from Asiatic pyogenic cholangitis, with a small fibrous left lobe with dilated ducts, who underwent left hepatic lobectomy.

The following microscopical features were listed for each section:

\section{SINGLE CELL THICK PLATES}

The presence of plates a single cell thick is now recognised as the hallmark of the normal arrangement in the adult. ${ }^{15}$ The plates are flanked by sinusoids on each side, and a departure from this arrangement is reported in diseased states and in young children. ${ }^{1617}$

\section{PLATES TWO CELLS THICK}

The replacement of the normal hepatocyte plates one cell thick by thickened plates, in which two cells are seen on section between sinusoids, has been observed in many conditions and represents the results of proliferation or an increase in the number of liver cells. (Figs. 1 and 2). ${ }^{16}$ This transformation affected either most liver plates, including those in the perivenous regions, or was confined to the periportal regions. The transformation affecting most liver plates was referred to as "diffuse hyper- 


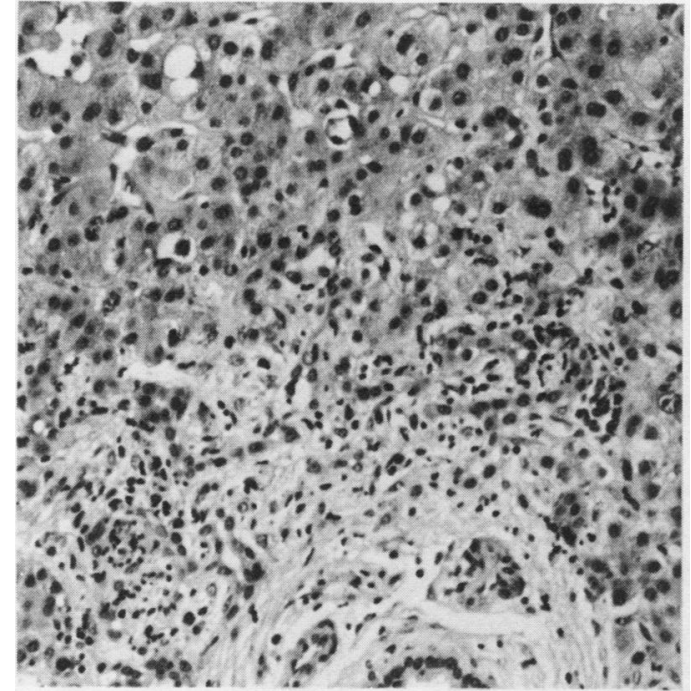

Fig 1 Wedge resection of liver showing diffuse thickening of hepatocyte plates. These are two cells thick near portal tracts and hepatic venous radicles. (Haematoxylin and eosin.) $\times 150$.

plasia" and that which affected the periportal regions as "periportal hyperplasia".

\section{FIBROSIS}

Fibrous tissue in portal tracts was considered to be increased when on section the tracts were about

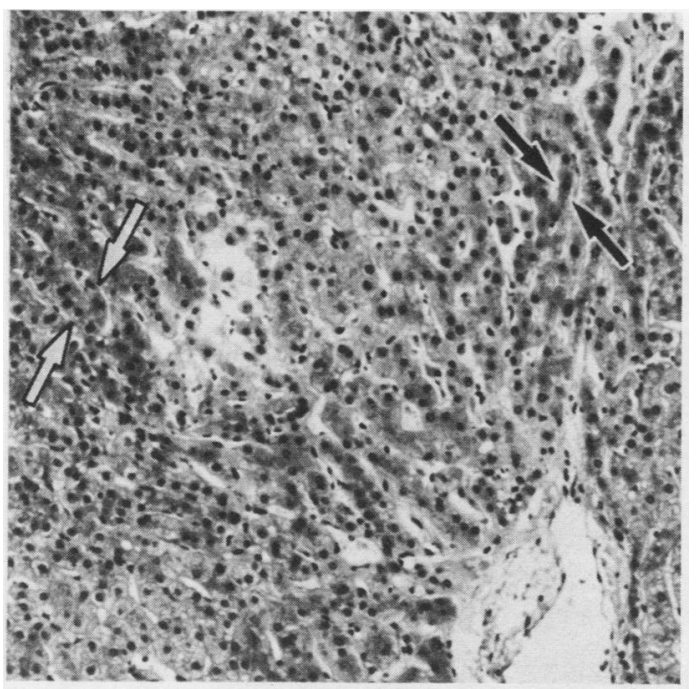

Fig 2 Wedge resection of liver showing plates two cells thick in periportal regions and plates one cell thick (normal) in perivenous regions. White arrows indicate sinusoid on each side of plate two cells thick, and black arrows indicate sinusoid on each side of plate one cell thick. (Haematoxylin and eosin.) $\times 150$. twice the normal diameter or greater and when they were composed predominantly of collagenous connective tissue on staining with Van Gieson's method. Sometimes the tracts were strikingly thickened by fibrous tissue and sections incorporated considerable lengths of such fibrous tracts. When transverse sections of more than one vessel or duct were included there appeared to be fibrous linkage between portal tracts (Fig. 3). It was regarded as important to identify fibrous tissue in all enlarged tracts as a complex of changes is usually noted in these regions in chronic bile duct obstiuction, often incorporating accumulations of lymphocytes and other mononuclear cells, exudative inflammation with oedema, organisation and proliferation of small ducts, as well as formation of fibrous tissue.

\section{RETAINED VASCULAR RELATIONS}

This referred to the identification of portal tracts and hepatic venous radicles in appropriate relation to each other. The main difficulty in the assessment of this was the definition of the hepatic venous radicle. In general, this structure was more oval than the adjacent sinusoids, lined by several clearly demarcated endothelial cells without Kupffer cells (Fig. 4), and well defined by impregnation with reticulin. Its position, usually equidistant from the centres of two portal tracts, helped to demarcate a lobule ${ }^{18}$ or an acinus, ${ }^{19}$ the arrangement found in normal liver tissue. The use of the term "lobule" therefore implies preservation of normal vascular relations and the term "nodule" refers to the changes in which normal vascular relations are not identified.

\section{CIRRHOSIS}

The consensus definition of cirrhosis was strictly adhered to, and the combination of diffuse thickening of plates without retention of normal vascular relations together with an increase and confluence of fibrous tissue was regarded as essential. ${ }^{20}$ If a normal vascular relation was not observed the change was categorised as comprising hyperplasia with formation of nodules and cirrhosis was diagnosed (Fig. 5). Cirrhosis was not diagnosed if a lobular structure was defined and normal relations between hepatic venous radicles and portal tracts were identified (Fig. 6).

It was possible to express a firm opinion in most instances about whether normal vascular relations were retained based on the identification of hepatic venous radicles. In one of our patients we were unable to recognise a normal vascular pattern, and cirrhosis was diagnosed. In all but one of the others hepatic venous radicles were found in most of the specimen and the spatial relation between these and the portal tracts was regarded as normal. In one 


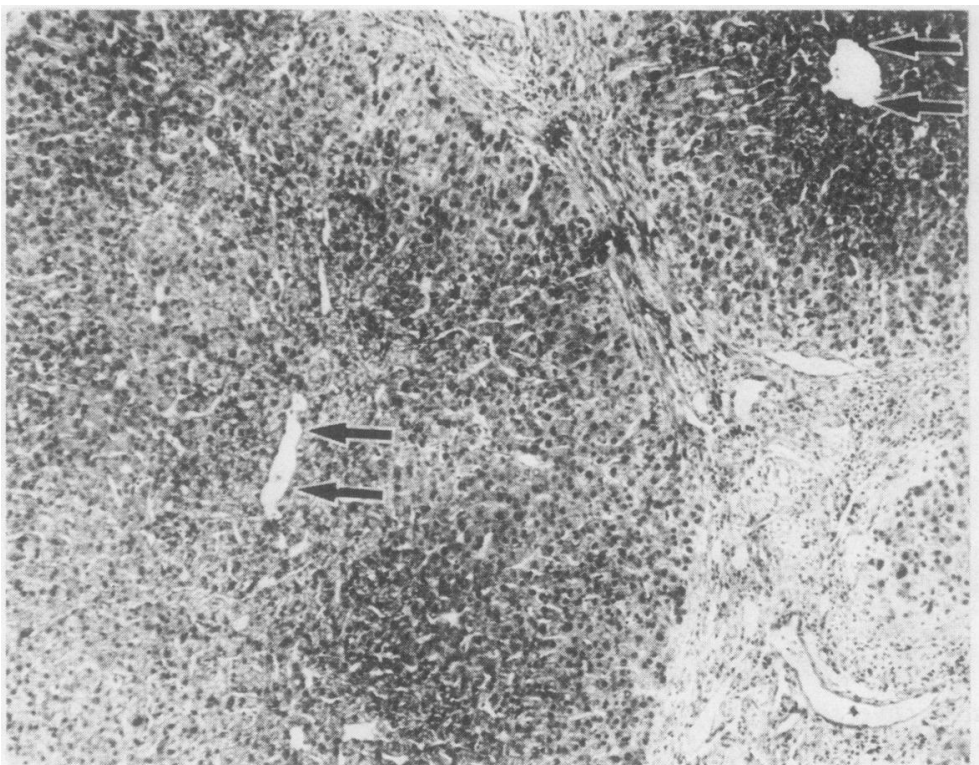

Fig 3 Wedge resection of liver with fibrous expansion of portal tracts giving appearance of fibrous linkage and isolation of parenchymal cells. Note survival of hepatic venous radicles (arrows). (Haematoxylin and eosin.) $\times 60$.

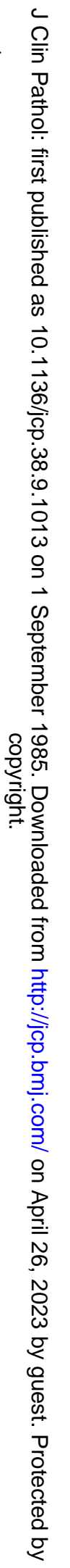

Fig 4 Extensive fibrosis with substantially normal relation of hepatic venous radicles to portal tracts from patient with portal hypertension. (Gordon and Sweet reticulin impregnation.) $\times 40$. 


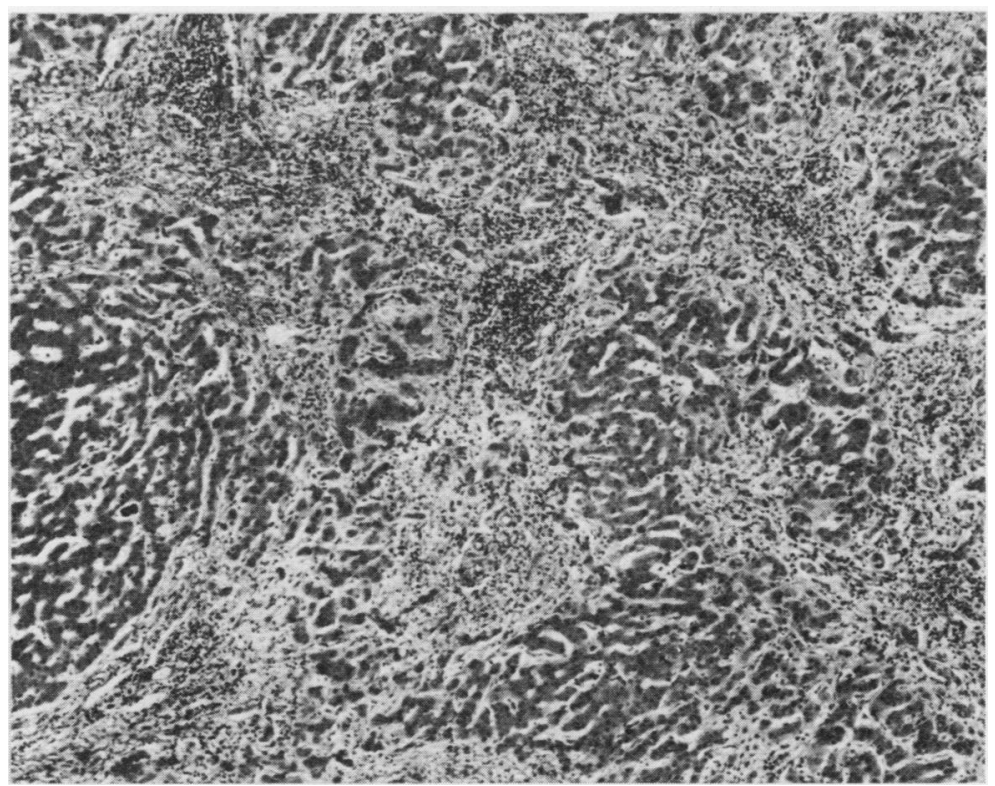

Fig 5 Confuent fibrosis with loss of vascular relations in wedge resection from patient with true cirrhosis. No hepatic venous radicles identified. (Haematoxylin and eosin.) $\times 60$.

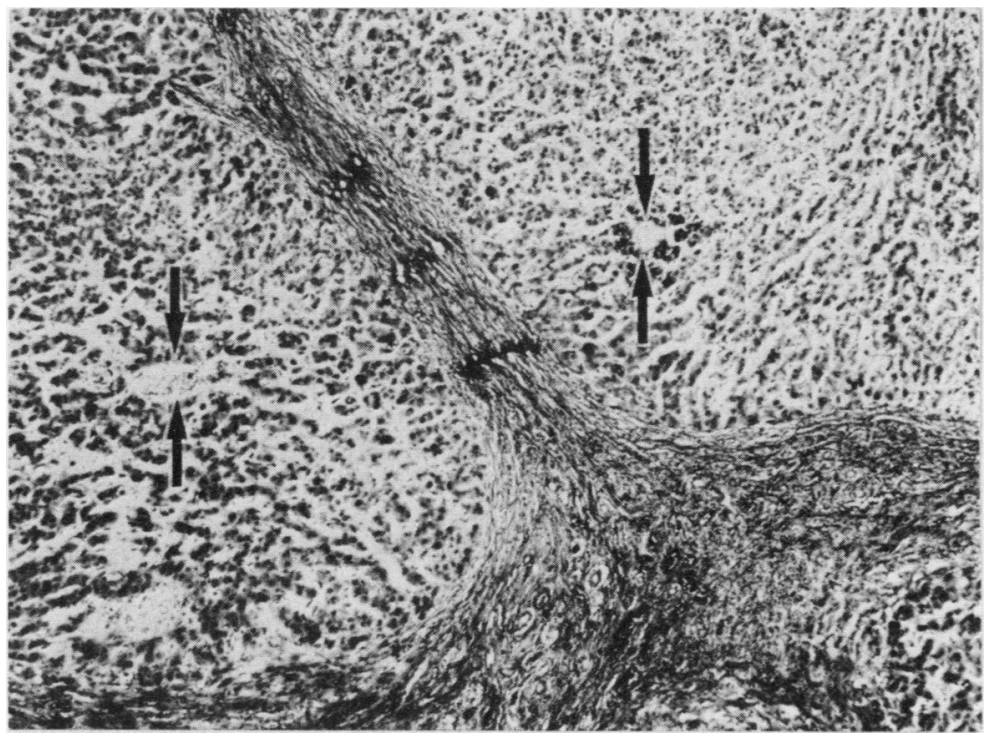

Fig 6 Necropsy section from patient with some survival of vascular relations. Hepatic venous radicles in some parts (arrows) were identified. (Long Elastic and Van Gieson) $\times 40$. 
instance part of the specimen contained vessels that might have represented venous radicles, but in other parts such structures were not observed. The patient was considered to have probably developed cirrhosis by the time she died from infection.

The microscopical findings indicated the presence of extensive fibrosis and diffuse hepatocyte hyperplasia in all specimens from patients with portal hypertension, but in 23 of the 25 instances vascular relations were, for the most part, retained. The clinical features of portal hypertension were not noted in two patients in whom the biopsy changes included diffuse hyperplasia and widely fibrosed portal tracts. These patients were 32 and 44 years of age, and we were not able to account for this discrepancy.

Hepatocyte hyperplasia and portal fibrosis were also noted in the 76 patients who were nonhypertensive, but these features were in general less pronounced than those encountered in the main group of patients suffering from portal hypertension. Only 23 of the 76 patients showed diffuse hepatocyte hyperplasia, and in 53 the thickened plates were confined to the periportal region. In all 76 the width of the portal fibrosis was estimated to be substantially less than that in the group with portal hypertension.

\section{Discussion}

The portal vein at the hilum of the liver was partly obstructed by tumour in three patients, and although we were not certain, we thought it reasonable to assume that this lesion was directly responsible for the development of splenomegaly. At any rate, we did not think it appropriate to include the hepatic microscopical changes in the analysis, although these were severe.

Gross disparity between liver lobes, with severe reduction by fibrosis of either right or left liver lobes was found in seven patients with signs of portal hypertension, and all had profound intrahepatic abnormalities in both liver lobes. A comparable degree of disparity was noted in eight patients in whom features of portal hypertension were absent. Although it is true that the disparity was found in a greater proportion $(7 / 25)$ of patients with features suggestive of portal hypertension than in those without $(8 / 76)$, the duration of disease in the group without was much shorter. On the other hand the intrahepatic features of hyperplasia and fibrosis in the patients with both disparity and portal hypertension were far more severe and extensive than these changes in patients with liver disparity but with no signs of portal hypertension. It seems, therefore, that reduction in size of one lobe cannot be regarded as a basis for splenomegaly or other signs of portal hypertension.
The structural changes of cirrhosis were considered to be present definitely in one patient and probably in a second. Hyperplastic nodules were separated by confluent abundant fibrosis, and the cirrhosis was similar structurally to some other forms of cirrhosis that are known to be associated with the development of portal hypertension. It should be emphasised, however, that the first of these two patients had a history of alcoholism as well as biliary tract disease, and, apart from micronodular cirrhosis, no other stigmata of chronic liver disease were found.

The main problem rests with the 23 other patients who showed clinical changes interpreted as resulting from portal hypertension. The liver changes in all 23 patients consisted of diffuse transformation to hepatocyte plates, two cells thick, with a generally retained normal relation between hepatic vein radicles and portal tracts accompanied by substantial portal fibrosis. Although evidence of hyperplasia was noted in the other group of patients in whom portal hypertension was not suggested, this was periportal only in 53 and diffuse in 23 and not usually associated with extensive fibrosis. The changes in the group without portal hypertension were not, therefore, as severe as those in patients with portal hypertension but also consisted of hepatocyte hyperplasia and portal fibrosis.

General questions arise in relation to these obser vations. The first is whether it is justifiable to regarde splenomegaly, with or without visible varices, as indicative of portal hypertension. In three instances actual measurements were made, and in six instances splenic tissue was available in which the characteristic microscopical changes of portal hypertension or fibrocongestive splenomegaly were found. Furthermore, laparotomy in 13 patients showed distended portal veins, and an intraabdominal surgical diagnosis of portal hypertension was made. In any case, the development of splenomegaly, with or without varices, is generally regarded as indicating portal hypertension in chronic biliary disease. ${ }^{2}$

The second question relates to the interpretation of hepatocyte plates two cells thick. The general consensus is that this change represents proliferation of hepatocytes, and this is suggested in many other conditions in which this is observed, such as diffuse nodular hyperplasia, ${ }^{21}$ cirrhosis, ${ }^{16}$ hepatic venous occlusion, ${ }^{22}$ and postnecrotic conditions. ${ }^{23}$ That this response is a common finding in long standing biliary disease is not unexpected, as it is clear that liver cells are capable of proliferating in the presence of duct obstruction ${ }^{24}$ and at the same time a continuous cellular destruction seems to proceed as a result of the extensive portal inflammation and ductal proliferation abutting on adjacent hepatocytes. 
Portal hypertension in chronic bile duct obstruction is probably seldom the result of cirrhosis, with its distorted vascular relations and its acknowledged structural irreversibility. In most instances the basis for the clinical features seems to be the combination of diffusely thickened hepatocyte plates and increased fibrous tissue in which a normal relation is maintained between portal tracts and hepatic venous radicles. This may be of importance in the assessment of such patients who develop features of portal hypertension, because the possibility of regression of the structural changes seems to be feasible and appropriate treatment may therefore be contemplated.

In one patient, however, part of the specimen was distorted and showed the appearance of cirrhosis and part showed retained vascular relations. The juxtaposition of these two states suggests that progression may occur, and although our series includes only one other patient with cirrhosis, the development of cirrhosis from bile duct disease, although unusual, must be regarded as possible. By the same token, qualitatively similar changes in patients without portal hypertension and with a shorter duration of disease suggest that portal hypertension may develop in time in patients with chronic bile duct obstruction.

It seems possible that the main component processes - that is, hyperplasia and fibrosis - may be corrected, provided normal basic vascular relations are preserved, as evidenced by the remodelling of hepatocyte plates two cells thick into single plates, which occurs in young children, ${ }^{17}$ and by the suggestion that collagen types I and III are labile."

In addition, the reported findings may help elucidate one of the paradoxes of chronic biliary tract disease and be of some importance in the therapeutic options open to patients who develop portal hypertension. Published findings agree that the development of "true" cirrhosis is a distinctly unusual complication in chronic biliary disease, amounting to a reported incidence of $8 \%$ in long standing disease ${ }^{78}$; yet a far higher incidence of patients attending hepatobiliary clinics seem to be treated for portal hypertension, and various forms of portal systemic anastomoses and biliary drainage are reported as sometimes being successful. ${ }^{12910}$

In most instances the structure of the liver, which is considered to be responsible for the fibrocongestive splenomegaly or portal hypertension, is not detailed and it is not possible, therefore, to determine whether the hepatic lesions include the changes now recognised as connoting cirrhosis which, in general, are considered to be irreversible. ${ }^{25}$ In some instances, indeed, the features regarded as indicating biliary cirrhosis clearly do not represent cirrhosis, as preservation of hepatic venous radicles is described and even illustrated..$^{13} 26$ This may be accounted for by the use of the term "cirrhosis" to represent increased fibrosis, a common accompaniment of chronic biliary disease. In fact, the term "biliary fibrosis" was at one time recommended, but this does not encompass the important aspect of hepatocyte proliferation. ${ }^{27}$

Evidence from experimental work 28 and the clinical experience referred to suggests that regression of structural changes in the liver is a possibility in most patients with portal hypertension in biliary tract disease. This may not be the case, however, when the vascular deformity and nodule formation of true cirrhosis are present, although this is distinctly uncommon. The possibility of regression of fibrosis must also be postulated if restitution is to be expected, and there is evidence that this may in fact occur in patients with excessive fibrous tissue in the liver as well as other organs. ${ }^{3031}$

Some drugs seem to effect a reduction in collagen types I and III, both of which are present in the liver, but the mechanism is not clearly understood. ${ }^{32}{ }^{33}$ It may be that collagenase, normally detected together with formative enzymes during synthesis of collagen, is present in excess in undefined circumstances or may be stimulated into activity with reduction of the inflammatory or proliferogenic processes attendant on chronic obstructive biliary tract disease.

We thank the Cancer Research Campaign for a grant given to $\mathrm{KW}$. We were given considerable technical help from the histopathology department under the direction of Professor NA Wright and $\mathrm{Mr}$ WF Hinkes of the department of medical illustration. Margaret Blount cut many sections and Agnes Gorrissen prepared many macrophotographs and also the typescript.

\section{References}

' Sedgwick CE, Poulantzas JK, Kune GA. Management of portal hypertension secondary to bile duct strictures. Ann Surg 1966;163:949-53.

${ }^{2}$ Cole WH, Ireneus C, Reynolds JT. Strictures of the common bile duct. Ann Surg 1955;142:537-51.

${ }^{3}$ Conn HO. Cirrhosis. In: Schiff L, ed. Diseases of the liver. 4 th ed. Philadelphia: J B Lippincott, 1975:833-939.

4 Doehlert CA, Baggenstoss AH, Cain JC. Obstructive biliary cirrhosis and alcoholic cirrhosis. Am J Clin Pathol 1955;25:902-14.

s Reynolds TB, Ito S, Iwatsuki S. Measurement of portal pressure and its clinical application. Am J Med 1970;49:649-57.

- Kelty RH, Baggenstoss AH, Butt HR. The relation of the regenerated liver nodule to the vascular bed in cirrhosis. Gastroenterology 1950;15:285-95.

' Gibson WR, Robertson HE. So-called biliary cirrhosis. Archives of Pathology 1939;28:37-48.

${ }^{8}$ Foulk WT, Baggenstoss AH. Obstructive or secondary biliary 
cirrhosis. In: Schiff L, ed. Diseases of the liver. 4th ed. Philadelphia: J B Lippincott, 1975:959-67.

' Adson MA, Wychulis AR. Portal hypertension in secondary biliary cirrhosis. Arch Surg 1968;96:604-12.

${ }^{10}$ Scobie BA, Summerskill WHJ. Hepatic cirrhosis secondary to obstruction of the biliary system. Am J Dig Dis 1965;10:13546.

"Rojkind M, Dunn MA. Hepatic fibrosis. Gastroenterology 1979;76:849-63.

${ }^{12}$ Popper H. Pathologic aspects of cirrhosis. Am J Pathol 1977;87:228-58.

${ }^{13}$ Cameron R, Bunton GL. Congenital biliary atresia. Br Med J 1960;ii: 1253-7.

14 Thaler MM, Gellis SS. Studies in neonatal hepatitis and biliary atresia. III. Progression and regression of cirrhosis in biliary atresia. Am J Dis Child 1968;116:271-9.

is Elias $\mathrm{H}$. A re-examination of the structure of the mammalian liver. I. Parenchymal architecture. Am J Anat 1949;84:31133.

${ }^{16}$ Scheuer PJ. Cirrhosis. In: MacSween RNM, Anthony PP, Scheuer PJ, eds. Pathology of the liver. Edinburgh: Churchill Livingstone, 1979:258-71.

17 Morgan JD, Hartroft WS. Juvenile liver. Archives of Pathology 1961;71:86-8.

${ }^{18}$ Matsumoto T, Komori R, Magara T, et al. A study of the normal structure of the human liver, with special reference to its angioarchitecture. Jikeikai Medical Journal 1979;26:1-40.

${ }^{19}$ Rappaport AM, Borowy ZJ, Lougheed WM, Lotto WN. Subdivision of hexagonal liver lobules into a structural and functional unit. Anat Rec 1954;119:11-33.

${ }^{20}$ Anthony PP, Ishak KG, Nayak NC, Poulsen HE, Scheuer PJ, Sobin LH. The morphology of cirrhosis. J Clin Pathol 1978;31:395-414.

21 Stromeyer FW, Ishak KG. Nodular transformation (nodular "regenerative" hyperplasia) of the liver. Hum Pathol "regenerative" hyperplasia) of the liver. Hum Pathol 1981;12:60-71.

${ }^{22}$ Parker RGF. Occlusion of the hepatic veins in man. Medicine 1959;38:369-402.

${ }^{23}$ Peters RL. Viral hepatitis: a pathologic spectrum. Am J Med Sci 1975;270: 17-31.

${ }^{24}$ Weinbren $\mathrm{K}$. The effect of bile duct obstruction on regeneration of the rat's liver. Br J Exp Pathol 1953;34:280-9.

${ }^{2 s}$ Conn HO, Groszmann RJ. The pathophysiology of portal hypertension. In: Arias IM, Popper H, Schachter D, Shafritz DA, eds. The liver biology and pathobiology. New York: Raven Press, 1982:821-48.

${ }^{26}$ Smith R, Sherlock S. Surgery of the gall bladder and bile ducts. 2nd ed. London: Butterworths, 1981:265.

${ }^{27}$ Desmet VJ. Cholestasis: extrahepatic obstruction and secondary biliary cirrhosis. In: MacSween RNM, Anthony PP, Scheuer PJ, eds. Pathology of the liver. Edinburgh: Churchill Livingstone, 1979:292-3.

${ }^{28}$ Rubin E, Popper H. The evolution of human cirrhosis deduced from observations in experimental animals. Medicine 1967; 46: 163-83.

${ }^{24}$ Popper H, Orr W. Current concepts in cirrhosis. Scand J Gastroenterol [Suppl] 1970;6:203-22.

${ }^{30}$ Bunton GL, Cameron R. Regeneration of liver after biliary cirrhosis. Ann NY Acad Sci 1963;111:412-21.

${ }^{31}$ Kershenobich D, Uribe M, Suarez GI, Mata JM, Perez-Tamayo $\mathbf{R}$, Rojkind M. Treatment of cirrhosis with colchicine. A double-blind 1979; 77:532-6.

${ }^{32}$ Nimni NE, Gerth N, Bavetta LA. Reversibility of penicillamine induced defect in collagen aggregation. Nature 1967;213:921-2.

${ }^{33}$ Rojkind M, Kershenobich D. Effect of colchicine on collagen albumin and transferrin synthesis by cirrhotic rat liver slices Biochim Biophys Acta 1975;378:415-523. 